\title{
Caracterización de estudiantes de la carrera de licenciatura en enfermería y sus percepciones sobre el consumo de drogas lícitas e ilícitas
}

\author{
Ligia Rojas Valenciano ${ }^{1}$ \\ Moacyr Lobo da Costa Junior ${ }^{2}$ \\ Gabriela Pereira Vasters ${ }^{3}$
}

\begin{abstract}
Se trata de un estudio cuantitativo, descriptivo y transversal. La población participante correspondió a 69 estudiantes que cursaban el quinto año de la carrera de Licenciatura en Enfermería. Se aplicó un cuestionario autoadministrado, previamente validado con estudiantes de enfermería que tenían características similares a los sujetos participantes. Este instrumento se dividió en cinco partes: información general, sociodemográfica, económica, personal, familiar y sociocultural. El estudio sugiere que el fenómeno de las drogas es multifactorial, está asociado a características sociodemográficas, económicas, personales, familiares y socioculturales. Entre las principales características del estudiantado están que es una carrera preponderantemente femenina, de clase media, con buen rendimiento académico, pero con inadecuados estilos de vida. Con relación al consumo de drogas, $44,9 \%$ ha consumido y consume actualmente bebidas alcohólicas, un porcentaje menor fuma cigarrillos y el 1,4\% fuma marihuana; además, no existe una conciencia clara de las consecuencias y conductas de riesgo a las que están expuestos al realizar dichas prácticas.
\end{abstract}

Descriptores: Estudiantes de Enfermería; Drogas Ilícitas; Transtornos Relacionados con Sustâncias.

\footnotetext{
${ }^{1}$ Enfermera, Doctor en Educación, Profesor Asociado, Escuela de Enfermería, Universidad de Costa Rica. E-mail: ligiarojas7@gmail.com

2 Estadístico, Doctor em Salud Publica, Profesor Asociado, Escola de Enfermagem de Ribeirão Preto, Universidade de São Paulo, Centro Colaborador de la OMS para el Desarrollo de la Investigación en Enfermería, Brasil. E-mail: mlobojr@eerp.usp.br.

${ }^{3}$ Estudiante de Doctorado, Escola de Enfermagem de Ribeirão Preto, Universidade de São Paulo, Centro Colaborador de la OMS para el Desarrollo de la Investigación en Enfermería, Brasil. E-mail: gabi_vastesr@yahoo.com.br.
}

Correspondencia:

Moacyr Lobo da Costa Junior

Universidade de São Paulo. Escola de Enfermagem de Ribeirão Preto

Av. Bandeirantes, 3900

Bairro Monte Alegre

CEP: 14040-902 Ribeirão Preto, SP, Brasil

E-mail: mlobojr@eerp.usp.br 


\title{
Caracterização de estudantes de licenciatura em enfermagem e suas percepções sobre o consumo de drogas lícitas e ilícitas
}

É um estudo quantitativo, descritivo e transversal. A população participante correspondeu a 69 estudantes que cursavam o quinto ano de Licenciatura em Enfermagem. Utilizou-se questionário autoadministrado, previamente validado com estudantes de enfermagem que cursavam outros anos do curso. Esse instrumento é dividido em cinco partes: informação geral, sociodemográfica, econômica, pessoal, familiar e sociocultural. O estudo sugere que o fenômeno das drogas é multifatorial, pois está associado a características sociodemográficos, econômicas, pessoais, familiares e socioculturais. Entre as principais características dos estudantes encontra-se: é uma carreira preponderantemente feminina, de classe média, com bom rendimento acadêmico, mas com inadequados estilos de vida. Em relação ao consumo de drogas, 44,9\% tinham consumido e consomem, atualmente, bebidas alcoólicas, outra porcentagem menor fuma tabaco e 1,4\% fuma maconha, porém, não existe consciência clara das consequências e condutas de risco a que estão expostos por realizar tais práticas.

Descritores: Estudantes de Enfermagem; Drogas Ilícitas; Transtornos Relacionados ao uso de Substâncias.

\section{Characterization of Students of the Nursing Degree Course and their Perceptions about Licit and Illicit Drug Consumption}

\begin{abstract}
This was a quantitative, descriptive, cross-sectional study. The participant population consisted of 69 students who were attending the fifth year of the Nursing Degree course. A self-administered questionnaire was applied, which was previously validated with a sample of nursing students that were attending other years of the course. The instrument was divided into five domains: general, sociodemographic, economic, personal, familiar and sociocultural information. The study evidence, that the phenomenon of drugs is multifactorial, is associated with sociodemographic, economic, personal, familiar and sociocultural characteristics. The students were predominantly female, of the middleeconomic class, with good academic performance, but had unhealthy life styles. Concerning drug consumption, $44.9 \%$ had consumed alcoholic beverages and consumed at the time of the study, another smaller percentage smoked tobacco and $1.4 \%$ smoked marijuana; in addition, a clear knowledge of risk behaviors and of the consequences to which they are exposed did not exist within this group.
\end{abstract}

Descriptors: Students, Nursing; Street Drugs; Substance-Related Disorders.

\section{Introducción}

Si bien es cierto que las drogas existen desde que existe el hombre, su evolución y el consumo evidente han ocasionado un serio problema de salud internacional. Investigando en la historia sobre este flagelo, se encontró que entre las drogas más antiguas utilizadas se encuentra el alcohol, descubierto a partir de la hidromiel, hace más de cuatro mil años, antes de Cristo por los Egipcios, quienes tenían estipulado su consumo para los escolares, la ración diaria era de tres panes de trigo y dos jarras de cerveza, que la madre tenía que llevar al niño a la escuela(1).

Aunado a lo anterior, la historia ha demostrado que la influencia del alcohol ha sido tan importante que sustituyó en los ritos religiosos, desde tiempos antiguos, a la sangre de animales utilizada para las consagraciones, por el vino; adjudicándole al mismo poderes mágicos, incomprensibles en aquella época para el hombre. 
Al igual que el alcohol, otras drogas de carácter ilícito, han sido descubiertas hace miles de años, por ejemplo los opiáceos que han sido mezclados con el tabaco y comercializados en el ámbito mundial; de ellos proceden la morfina y la heroína. Por otro lado, la cocaína también ha sido utilizada para llevar a cabo ritos religiosos; para los incas representaba la divinidad. Otras drogas son las anfetaminas, el cannabis, los alucinógenos, el tabaco, las drogas inhalables y las sintéticas, productos creados por el hombre en las más diversas culturas y épocas, para diferentes fines, los que ha conducido a la sociedad, por múltiples factores, a enfrentar una serie de problemas de drogadicción y riesgo para las generaciones futuras.

Actualmente, los países tienen una gran preocupación y han declarado el consumo de drogas como un problema de salud pública y causante de morbilidad y mortalidad, accidentes de tránsito, accidentes laborales, suicidios, homicidios y violencia doméstica(2).

Debido a lo anterior, se resalta la importancia de fomentar y fortalecer el conocimiento del fenómeno de las drogas que permita un mejor entendimiento $y$ control del consumo y abuso, especialmente en aquellas poblaciones que constituyen objetivos de intervención, las cuales están dirigidas especialmente para enfrentar este flagelo que está afectando a los niños, adolescentes y adultos jóvenes. El fenómeno de las drogas, en Costa Rica, adquiere gran importancia en la investigación e información a partir de los años noventa. Es el Instituto de Alcoholismo y Farmacodependencia (IAFA) quien encabeza la mayor parte de las investigaciones, aproximadamente el 57\%, seguido por la Universidad de Costa Rica con un $11 \%$ hasta el año 1995. En adolescentes se había investigado en un $14,7 \%$ para $1997^{(3)}$.

En la Universidad de Costa Rica (UCR) un estudio sobre la prevalencia presenta las características de los estudiantes de la Facultad de Farmacia y sus actitudes, conocimientos y prácticas hacia el consumo de alcohol y otras drogas ${ }^{(4)}$. Otro estudio de la Universidad de Costa Rica encontró que la droga más utilizada es la cafeína, reportándose en una cuarta parte de los estudiantes el síndrome de abstinencia a la misma(5). Como lo demuestran los estudios antes presentados, la drogadicción es un fenómeno de gran impacto a nivel social, cultural, económico, político y legal, y abre paso para elaborar diversos mitos y creencias alrededor de los diferentes grupos poblacionales; por ejemplo, es común pensar que el grupo de los profesionales en salud son inmunes al uso y abuso de drogas por su formación y más bien luchan contra el flagelo de las drogas; pero también debe considerarse que tienen mayor acceso a la obtención de drogas por estar en contacto con muchas de ellas. Por esta razón es muy importante la información y formación multidisciplinar en los currículos de las carreras de salud que brindan las universidades en materia de drogas, ya que los programas y proyectos existentes en estas instituciones educativas no son suficientes para lograr una verdadera introyección y concientización en los estudiantes.

El ambiente y las características propias del estudiante universitario, según algunos estudios realizados en la UCR conjuntamente con el IAFA, no favorecen en ningún sentido la disminución significativa del fenómeno de las drogas. Por tal motivo, se considera importante implementar dentro del plan de estudios de las carreras, la temática sobre el fenómeno de las drogas desde una perspectiva de salud internacional que le brinde al estudiante las herramientas cognitivas sobre la drogadicción para sí mismo y para brindar la atención a las personas en el ámbito de la promoción y prevención.

Este artículo presenta un análisis de las características sociodemográficas, personales, familiares y económicas de los estudiantes de quinto año de la Licenciatura en Enfermería de la Universidad de Costa Rica y su percepción sobre el fenómeno de las drogas.

\section{Materiales y Métodos}

Esta investigación forma parte de los objetivos planteados en el proyecto de investigación No. 200 titulado "Factores sociales, culturales, personales, familiares y económicos asociados al fenómeno de las drogas en estudiantes de la carrera de Licenciatura en Enfermería: un estudio desde la perspectiva de la Salud Internacional", de la Escuela de Enfermería de la Universidad de Costa Rica, presentado y aprobado por la Vicerrectoría de Investigación de esa universidad.

Se trata de un estudio cuantitativo, descriptivo y transversal. La población participante correspondió a 69 estudiantes que cursaban el quinto año de la carrera de Licenciatura en Enfermería; por ser una población pequeña, se decidió trabajar con el total. Se aplicó un cuestionario estructurado autoadministrado, el cual fue elaborado y previamente validado en 2007, con una muestra del $10 \%$ de estudiantes de enfermería que tenían características similares a los sujetos participantes que cursaban otros años de la carrera. El cuestionario utilizado se dividió en cinco partes: información sociodemográfica, económica, personal, familiar y sociocultural. 
Después de recolectar los datos informados en el instrumento, se elaboró una base de datos utilizando el paquete estadístico "Statistical Package for Social Science"; se procesó la información mediante el uso de estadística descriptiva. Finalmente, se procedió hacer una comparación y a analizar los datos obtenidos y la teoría seleccionada para esta investigación.

Con respecto a los fundamentos éticos, en consonancia con las normas del comité de ética de la Vicerrectoría de Investigación de la Universidad de Costa Rica, se elaboró y aplicó el término de consentimiento informado que contenía: descripción del estudio, objetivo, beneficios y aseguraba al participante la confidencialidad de la información.

\section{Resultados}

\section{Factores sociodemográficos}

En factores sociodemográficos se observó que la población de quinto año está concentrada en el intervalo de edad de 21 a menos de 25 años, con un 78,2\%; además, el $87 \%$ eran mujeres, solteras y el $68,1 \%$ informó ser católico. Las variables demográficas señalan que durante el tiempo lectivo el $68,1 \%$ vivía con sus padres y tan sólo un $14,4 \%$ vivían con compañeros y amigos.

\section{Factores económicos}

En relación a los factores económicos los resultados reflejaron que el estudiantado en un $79,7 \%$ estaba trabajando; sin embargo, sólo el 2,9\% era del tipo remunerado registrado. Con respecto a las horas que trabajan, el 8,7\% trabajaba menos de 4 horas por día. También se les preguntó sobre las becas otorgadas por la universidad, 92,8\% declaró que habían recibido una beca, y el 75,3\% era del tipo de 8 a 11 (en el tipo de beca 8 se exonera al estudiante de un porcentaje proporcional a sus ingresos del pago de créditos matriculados, en la beca 11 se apoya al estudiante económicamente y de forma proporcional). Señala el estudiantado que los ingresos económicos no son suficientes $(58,8 \%)$, siendo el padre quien realiza el aporte $(68,1 \%)$ y la madre en un 43,5\%; además estos ingresos, en un $85,5 \%$ eran provenientes del salario. Por lo cual, una de las perspectivas futuras que tienen los estudiantes es realizarse profesional y financieramente $(98,6 \%)$.

\section{Factores personales}

Con relación a las diversas actividades que realizan los estudiantes se evidenció que un $42 \%$ asiste a la iglesia una vez por semana y el $43,5 \%$ no lo hace ninguna vez. El $46,4 \%$ realiza diversas actividades culturales y un $34,8 \%$ (53 estudiantes) visita bares, tabernas, salones de baile y discotecas. Según el aspecto académico, el 71\% (49 estudiantes) mencionaron estar satisfechos con la elección de su carrera. Por otro lado, su desempeño en la universidad es muy bueno, suelen estudiar 4 horas diarias $(65,2 \%)$; esto se ratifica en el porcentaje de aprobación de cursos, donde en el último semestre el $98,6 \%$ aprobó todas las materias matriculadas. La biblioteca es un sitio muy frecuentado por ellos $(53,6 \%)$. Otro aspecto que se encontró fue que el 40,6\% nunca iban al Centro de Tecnología en Enfermería (CATE) y tan sólo un $42,0 \%$ asistía con poca frecuencia. Otro lugar al que refieren nunca visitar es la Asociación de estudiantes $(68,1 \%)$.

Una variable interesante que se investigó fue sobre las relaciones interpersonales, al respecto el $88,4 \%$ manifestó, que no tiene dificultad para hacer nuevos amigos, ni se han sentido relegados por el grupo de amigos de su misma edad; además, el $87 \%$ declaró no ser fácilmente influenciable, y el $100 \%$ señaló que no harían cualquier cosa para ser aceptado en un grupo. Por otro lado, el 92,8\% expresó que sus opiniones son tomadas en cuenta.

Con respecto al consumo de drogas lícitas e ilícitas; el 58\% manifestó haber consumido drogas. El 39,1\% expresó que no le llamaba la atención el utilizar drogas, el $31 \%$ sabía los efectos de las drogas y un $30,4 \%$ expresó que no necesitaba la droga o no la utilizó por razones de principios y valores morales. Entre los que dijeron haber consumido drogas, el 17,4\% señaló que fueron los amigos y compañeros quienes las ofrecieron. La edad en que le ofrecieron drogas estuvo mayoritariamente en el rango de edad de 15 a menos 17 años para un $8,6 \%$, seguido de 17 a menos de 20 años con el 5,8\%.

Con respecto a los estudiantes que consumen drogas, específicamente bebidas alcohólicas, el 29\% (20 estudiantes) manifestó que alguna vez sintieron que deberían disminuir la cantidad de bebida o parar de ingerir alcohol y solamente el 4,3\% (3 estudiantes) señaló que se sentía culpable por la manera con que acostumbraba ingerir alcohol.

Además, el 20,3\% opinó que le era indiferente el uso de tabaco y el $42 \%$ el uso de alcohol en reuniones sociales (fiestas, actividades religiosas, familiares, reuniones formales e informales, entre otras). No obstante, el 84,1\% expresó estar en desacuerdo con el uso de drogas ilícitas en este tipo de eventos. 
El estudiantado manifestó que la razón por la que se usa drogas, incluyendo alcohol y tabaco, dentro del ámbito social es para relacionarse con otras personas $(34,8 \%)$, por diversión o placer $(50 \%)$, para acompañar a su pareja o amigos (37,7\%), y en menor porcentaje con un $27,5 \%$ el pertenecer a un grupo.

Se exploró también si los estudiantes habían estado sometidos a situaciones de riesgo; al respecto el 23,2\% respondió que tenían una alta probabilidad de estar expuestos al consumo de bebidas alcohólicas, el $26,1 \%$ de consumir alimentos fritos, el 20,3\% tenía alta probabilidad de estar expuesto al contacto con personas con enfermedades infectocontagiosas y el $18,8 \%$ de llevar una vida sedentaria. Por otro lado, mencionaron, en un alto porcentaje, que tenían baja probabilidad $(88,4 \%$ y $91,3 \%$ respectivamente) de usar cocaína, crack, éter o inhalar cualquier tipo de solvente.

El 50,7\% opinó que los amigos le ofrecieron alcohol, el $26,1 \%$ tabaco y el $10,1 \%$ marihuana. Es importante resaltar que el $21,7 \%$ (15 estudiantes) refieren que un familiar le ofreció alcohol, el 4,3\% tabaco y un $1.4 \%$ la marihuana. El motivo que los impulsó a usar drogas es pasarlo bien $(44,9 \%)$ y el $37,7 \%$ declaró que era para saber cómo es. El 15,9\% abandonó el uso por problemas de salud y el $14,5 \%$ porque no les llamó la atención seguirlo haciendo.

La edad en que se le ofreció drogas, se concentra en los intervalos 13 a menos de 16 años (28,9\%) y en de 16 a menos de 18 años con 26,1\%; estos mismos intervalos se destacan en la edad de inicio de uso con un $14,5 \%$ para el primer intervalo y $24,6 \%$ para el segundo, de 18 a menos de 20 años, un 17,3\%. Con respecto al abandono del uso de drogas incluyendo alcohol y tabaco, en los tres intervalos anteriores, se encontró que el 2.9\% lo abandonó. En relación con el tabaco, se encontró el porcentaje de $18,8 \%$ en el intervalo de 16 a menos de 18 años; la edad de inicio es igual en porcentaje (4,3\%) para todos los intervalos; y con respecto al abandono del tabaco, la opinión de los estudiantes se concentró en el intervalo de 20 años y más con un 7,2\%. La edad en que se le ofreció marihuana tenía un mayor porcentaje en el intervalo de 16 a menos de 18 años (7,2\%); no obstante, el inicio de uso para ese mismo intervalo de edad es de tan sólo 1,4\%, porcentaje igual al del abandono. Se destaca también que el 2,9\% la abandona a la edad de 20 años y más.

Al hacer referencia al uso actual de drogas, los estudiantes $(40,6 \%)$ mencionaron que fue ofrecida por los amigos y un $14,5 \%$ por sus familiares. El motivo para consumir alcohol actualmente, en un $36,2 \%$ era para pasarla bien; el $74,4 \%$ la consumió con amigos y compañeros. La frecuencia de uso se concentró en un $34,8 \%$ en el intervalo de menos de una vez por semana. El estudiantado opinó, en un 30,4\%, que el uso frecuente de alcohol no interfiere con diversas actividades que se realizan como alimentación, sueño, estudio, trabajo, relaciones afectivas y sociales $y$ el desempeño sexual. El 27,5\% expresó que no ha pensado en dejar de consumir alcohol y el 36,2\% no ha buscado ayuda para dejar de consumirlo. Con respecto al tabaco y otras drogas no hay porcentajes significativos en cuanto al uso.

\section{Factores familiares}

El mayor porcentaje de escolaridad de los padres se concentró en la variable "universitaria" con un 36,2\%, seguido de primaria completa para un $23,2 \%$.

Con respecto al uso de drogas en la familia incluyendo alcohol y tabaco, refieren que el 69,6\% consume. Además, en los últimos 12 meses, algún familiar ha consumido a causa de problemas familiares, con amigos o en el trabajo, y en los últimos cinco años algunos familiares $(8,7 \%)$ se ha visto relacionado en accidentes de tránsito después de haber usado drogas. El 44,9\% mencionó que se ingiere alcohol en actividades familiares y religiosas en las que participa; sin embargo, con respecto a la frecuencia de uso el 23,2\% destacó que lo hace con poca frecuencia, únicamente el 17,4\% lo señaló como frecuente y un $8,7 \%$ muy frecuente; en relación con el tabaco, este es usado por el $24,6 \%$ y de forma frecuente por un 13\%; tan sólo un $2,9 \%$ refieren usar drogas ilícitas raras veces en este tipo de actividad.

Las relaciones interpersonales han sido afectadas con el uso frecuente de drogas, incluyendo alcohol y tabaco: en la familia $(36,2 \%)$, laboral $(20,3 \%)$ y con la pareja fue mencionado por el $18,8 \%$. El $58 \%$ mencionó que los familiares no buscaron ayuda para disminuir el uso.

Finalmente, en este factor, se preguntó sobre quien hacía uso no médico y frecuente de drogas tres veces por semana como mínimo; los estudiantes contestaron que los tíos ingieren alcohol $(26,1 \%)$, seguido de hermanos $(15,9 \%)$, otros parientes $(13 \%)$, y el padre con un $11,6 \%$. Con respecto al tabaco, tanto tíos como hermanos fumaban en un $11,6 \%$, seguido por el padre con un $8,7 \%$. Referente a la marihuana solamente los 
hermanos la fumaban con un 4,3\%. Para el resto de drogas su respuesta fue negativa.

\section{Factores socioculturales}

En este factor se preguntó sobre la comodidad que posee el lugar donde vive actualmente. Ellos respondieron que se encuentran muy adaptados (69,9\%); únicamente el $1,4 \%$ se encuentra inadaptado. En relación al consumo de drogas, ellos opinaron en un 33,3\% que sus amigos hacen uso no médico de ellas; además, aceptan su uso, incluyendo alcohol y tabaco, en el ámbito social (44,9\%), siendo poco aceptado o inaceptable por el 49,3\%. Con respecto al consumo de drogas por parte de sus amigos, el 75,4\% (52 estudiantes) expresó que ingieren alcohol, el $62,3 \%$ (40 estudiantes) fumaban tabaco y el $17,4 \%$ (12 estudiantes) fumaban marihuana; para el resto de drogas el porcentaje no fue significativo.

\section{Discusión}

La población estudiantil de enfermería que estaba finalizando la carrera (quinto año) era una población joven, con predominio del sexo femenino, de clase media, un porcentaje importante $(68,1 \%)$ no eran practicantes activos de la denominación religiosa a la que declararon pertenecer. Entre las prácticas sociodemográficas que realizaban, el 44,9\% participaba en eventos religiosos familiares en donde se consumía alcohol y tabaco, drogas que son social y culturalmente aceptadas desde tiempos muy antiguos, y formaron parte de los rituales religiosos de aquellas épocas. Además, un porcentaje importante (68.1\%) vivía con sus padres, tan sólo el $14,4 \%$ vivía con compañeros y amigos. Esto es positivo en términos de convivencia en donde los valores y principios es la dinámica que suele predominar en el ambiente familiar, por lo cual se considera un factor protector en esta población estudiantil.

En relación a los aspectos económicos a los que hizo referencia el estudiantado, cabe destacar, que los ingresos económicos no eran suficientes para solventar sus gastos, por tal motivo, algunos de ellos y ellas trabajaban alrededor de cuatro horas al día, siendo este empleo en muchas ocasiones no registrado. Además, para hacerle frente a sus estudios solicitaban becas a la universidad, congruentemente con sus bajos recursos económicos gozan de becas altas ( 8 y 11 ).

Es comprensible en este grupo de edad, el querer compartir con el grupo de iguales, amistades y compañeros, en ambientes diversos; sin embargo, al visitar los sitios, antes mencionados, se exponen al consumo desde un refresco natural hasta drogas lícitas e ilícitas.

Al respecto de las amistades, estas tienen un papel importante porque son quienes ofrecen todo tipo de drogas incluyendo alcohol y tabaco. Es evidente que la edad de inicio de consumo mayoritariamente estuvo en un rango de edad de 15 a menos de 17 años; sin embargo, las estadísticas de otros estudios muestran que este rango de edad está disminuyendo. O sea, que son los niños y niñas en edad escolar los que se están iniciando en el consumo de drogas ${ }^{(6-7)}$.

Entre las drogas que se consumen, las bebidas alcohólicas tienen el primer lugar, seguido por el tabaco. El consumo del alcohol toma real importancia en la población universitaria, debido a que la pauta de consumo en la adolescencia y juventud, aumenta tanto en número de bebidas, como en su grado alcohólico. Los jóvenes en su ambiente universitario tienen mayor accesibilidad de obtener y consumir bebidas alcohólicas.

Entre las razones por las cuales se consume drogas incluyendo alcohol y tabaco, el estudiantado en enfermería lo hace por diversión o placer y por acompañar a la pareja o amigos. Motivos congruentes con el tipo de actividad que manifestaron realizar anteriormente. Al respecto señala un comentario de Valverde y Camacho que "Para nadie es un secreto que en la sociedad costarricense hay cantidad de bares, restaurantes $y$ cantinas en pueblos y ciudades, donde se expende licor indiscriminadamente, mientras los consumidores tengan potencial económico para adquirirlo, no importa si son menores de edad aunque esto sea castigado por ley. De esta manera se gestan centros de reunión donde se dan los primeros pasos en la vía del alcoholismo"(8).

Es interesante señalar con respecto al uso actual de drogas en este grupo de estudio, especialmente con el uso de bebidas alcohólicas, que el estudiantado no tiene conocimiento claro de las situaciones de riesgo a que están expuestos, ni a las implicaciones futuras sobre su trabajo, sus relaciones familiares, y en general en el contexto donde se relacionan; esto se evidenció en sus respuestas sobre el uso, en donde manifestaron que lo ingieren para pasarla bien, que el uso frecuente de alcohol no interfiere con sus actividades y no piensan en dejar de consumirlo.

Con respecto a los factores familiares los datos reflejaron que el estudiantado proviene en su mayoría de familias de clase media en donde sus padres tienen un alto porcentaje de alfabetización. No obstante, se evidenció el uso, dentro de sus familias, de drogas 
incluyendo alcohol y tabaco; este aspecto influye en la dinámica familiar afectando las relaciones interpersonales en la familia, en el trabajo y con la pareja; además, están expuestos a accidentes de tránsito después de haber usado drogas. Cabe resaltar que en el 14,5\% de los casos, ha sido un familiar quien ofreció drogas al estudiantado. En este sentido, un estudio multicéntrico encontró que los jóvenes entrevistados indicaron los factores psicosociales y no los biológicos o genéticos para explicar la causa de los problemas del consumo de drogas. También se identificó que las familias son un factor importante en la prevención del consumo de $\operatorname{drogas}^{(9)}$.

Muchos de los elementos que influyen para que se presente esta problemática de la drogadicción, se genera en la familia, ya sea por sobreprotección excesiva o el desinterés, por la falta de valores morales y espirituales, por la carencia o exceso de recursos económicos, entre otros aspectos, y por la carencia de un proyecto de vida familiar(8).

Finalmente, dentro de los factores socioculturales, cabe destacar que la población participante expresó que sus amigos hacen uso no médico de drogas. En relación con este tópico, un estudio sobre el consumo de benzodiacepinas, en una muestra nacional de 1.186 estudiantes y 197 adolescentes consumidores de drogas, demostró que el 3\% de los adolescentes estudiantes refirió haber consumido tranquilizantes en la vida, mientras que, en los consumidores de drogas, de 2 a 3 de cada diez, refirió haber consumido este tipo de sustancias en la vida(10). La edad promedio de inicio de consumo fue de 14 años. Por la capacidad adictiva de las benzodiacepinas, el consumo de estas sustancias en los jóvenes puede tener importantes implicaciones en su desarrollo y bienestar.

\section{Conclusiones}

En síntesis puede decirse que el presente estudio sugiere que el fenómeno de las drogas es multifactorial y su consumo tiene distintos significados y motivaciones para los distintos grupos de personas; también varía en su tipo y gravedad en las consecuencias mundiales, sociales, familiares $y$ personales que pueden producir.

Fue posible observar que los estudiantes conviven con amigos o familiares, los cuales en su mayoría consumen bebidas alcohólicas y fuman tabaco, siendo los amigos las personas que los han inducido a consumir esas sustancias. Los motivos que mueven al estudiantado a consumir alcohol son: sentirse bien, divertirse, por placer, para acompañar a la pareja y para pertenecer a grupos de iguales; consideran que esto no interfiera en su proyecto de vida, ni en sus relaciones familiares o académicas. Delante de esto, es importante que la universidad promueva espacios de discusiones entre los estudiantes como prevención al uso de riesgo tanto de alcohol como de otras substancias.

No obstante, es responsabilidad como profesionales, desde la academia, capacitar al personal docente, y motivar la participación estudiantil en espacios socioeducativos que promueven un cambio de conducta social para este fenómeno; y, de ese modo, puedan estar verdaderamente preparados para entrar al mundo laboral y ser parte del equipo de salud que discute e interviene en la problemática del alcohol y de otras drogas, en la comunidad en general.

\section{Agradecimientos}

Agradecemos a la Comisión Interamericana para el Control del Abuso de Drogas/CICAD de la Secretaria de Seguridad Multidimensional/SSM de la Organización de los Estados Americanos/OEA, la Secretaria Nacional de Políticas sobre Drogas/SENAD do Gabinete de Seguridad Institucional/Brasil, la Escuela de Enfermería de Ribeirao Preto de la Universidad de Sao Paulo y Centro Colaborador de la Organización Mundial de la Salud para el Desarrollo de la Investigación en Enfermería, la población representada en los estudios de investigación, bien como a las autoridades de las universidades representadas por los participantes del Programa En-Line de Especialización en Investigación sobre el Fenómeno de las Drogas PREINVEST, periodos 2005, 2006, 2007 y 2008.

\section{Referencias}

1. Pascual Arriazu J, Rubio Valladolid G. Historia General de las drogas. In: Santos D. Manual de drogodependencias para enfermeria. Madrid; 2002.

2. Jiménez RF. Síndromes de intoxicación y abstinencia a drogas psicoactivas: recomendaciones para su manejo. San José: IAFA; 2000.
3. Cascante A. Investigaciones sobre alcoholismo y farmacodependencia: Costa Rica. San José: IAFA; 1997.

4. Acuña, O, Amador G, Badilla B, Jiménez F. Consumo de sustancias psicotrópicas en los estudiantes de la Facultad de Farmacia de la Universidad de Costa Rica. Rev Cienc Soc $1992 ;(60): 63-72$. 
5. Badilla B, Barquero M. Prevalencia del uso de estimulantes en los estudiantes de medicina y farmacia de la Universidad de Costa Rica. Rev Cienc Soc. 1993; (61):121-9.

6. Jinez LJ, Souza JRM, Pillon Sandra Cristina. Uso de drogas e fatores de risco entre estudantes de ensino médio. Rev. LatinoAm. Enfermagem. 2009;17(2):246-52.

7. Pillon SC, O’Brien B, Piedra KAC. La relación entre el uso de drogas y comportamiento de riesgo entre estudiantes Brasileños. Rev. Latino-Am. Enfermagem. [internet]. 2005. [acceso en: 05 deciembre 2009]; 13 (especial). Disponible em: http://www. scielo.br/pdf/rlae/v13nspe2/pt_v13nspe2a11.pdf
8. Valverde L, Camacho D. Consumo de drogas. Rev Cienc Soc. 2003;(099):1-7.

9. Silva J, Ventura CAA, Vargens OMC, Loyola CMD, Albarracín DGE, Diaz J, et al. Illicit drug use in seven Latin American countries: critical perspectives of families and familiars. Rev. Latino-Am. Enfermagem. 2009;17(spe):763-9.

10. Sandí LE, Díaz AA. Consumo de Benzodiacepinas en adolescentes costarricenses. Farmacos.1996;9(1):31-7. 